\title{
Computer-Aided Drug Design Guided by Hydrogen/Deuterium Exchange Mass Spectrometry: A Powerful Combination for the Development of Potent and Selective Inhibitors of Group VIA Calcium-Independent Phospholipase $\mathbf{A}_{2}$
}

\author{
Varnavas D. Mouchlis, ${ }^{+*}$ Christophe Morisseau, ${ }^{\ddagger}$ Bruce D. Hammock, ${ }^{\ddagger}$ Sheng Li, ${ }^{\S}$ J. Andrew \\ McCammon, ${ }^{+*}$ and Edward A. Dennis ${ }^{+*}$ \\ ${ }^{\dagger}$ Department of Pharmacology and Department of Chemistry and Biochemistry, School of Medicine, University of \\ California, San Diego, La Jolla, California 92093-0601, USA, \\ ${ }^{\ddagger}$ Department of Entomology and Nematology and UC Davis Comprehensive Cancer Research Center, University of \\ California, Davis, CA 95616, USA, and \\ ${ }^{\S}$ Department of Medicine, University of California, San Diego, La Jolla, California 92093-0601, USA \\ Corresponding Authors \\ *V.D.M.: e-mail, vmouchlis@gmail.com \\ *J.A.M.:, e-mail: jmccammon@ucsd.edu \\ *E.A.D.: e-mail, edennis@ucsd.edu; phone, +1 8585343055.
}

\begin{abstract}
Potent and selective inhibitors for phospholipases $A_{2}\left(P L A_{2}\right)$ are useful for studying their intracellular functions. PLA $\mathrm{PL}_{2}$ enzymes liberate arachidonic acid from phospholipids activating eicosanoid pathways that involve cyclooxygenases (COX) and lipoxygenases (LOX) leading to inflammation. Anti-inflammatory drugs target COX and LOX; thus, PLA 2 can also be targeted to diminish inflammation. This paper describes the employment of enzymatic assays, hydrogen/deuterium exchange mass spectrometry (DXMS) and computational chemistry to develop $\mathrm{PLA}_{2}$ inhibitors. Beta-thioether trifluoromethylketones (TFKs) were screened against human GVIA calcium-independent, GIVA cytosolic and GV secreted PLA ${ }_{2} \mathrm{~S}$. These compounds exhibited inhibition towards Group VIA calcium-independent PLA (GVIA iPLA $_{2}$ ), with the most potent and selective inhibitor 3 (OTFP) obtaining an $X_{1}(50)$ of 0.0002 mole fraction ( $\left(C_{50}\right.$ of $110 \mathrm{nM}$ ). DXMS binding experiments in the presence of OTFP revealed the peptide regions of GVIA iPLA 2 that interact with the inhibitor. Molecular docking and dynamics simulations in the presence of a membrane were guided by the DXMS data in order to identify the binding mode of OTFP. Clustering analysis showed the binding mode of OTFP that occurred $70 \%$ during the simulation. The resulted 3D complex was used for docking studies and a structure-activity relationship (SAR) was established. This paper describes a novel multidisciplinary approach in which a 3D complex of GVIA iPLA 2 with an inhibitor is reported and validated by experimental data. The SAR showed that the sulfur atom is vital for the potency of beta-thioether analogues, while the hydrophobic chain is important for selectivity. This work constitutes the foundation for further design, synthesis and inhibition studies in order to

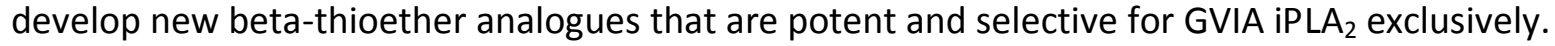

Keywords: Phospholipase $A_{2}$, trifluoromethylketones, thioether, DXMS, docking, MD simulations, structure-activity relationship. 


\section{Introduction}

Phospholipase $A_{2}\left(P L A_{2}\right)$ constitutes a superfamily of enzymes that is implicated in many biological pathways because of its unique ability to catalyze the hydrolysis of the ester bond at the $s n-2$ position of membrane phospholipid molecules $[1,2]$. The products of their catalytic action include arachidonic acid (AA) and other free fatty acids as well as lysophospholipids (LP). $A A$ and LP are metabolic precursors of numerous metabolites involved in inflammatory conditions including prostaglandins (PG), leukotrienes (LT), and platelet-activating factor (PAF) [3-5]. The PLA family includes six main types: cytosolic $\left(\mathrm{CPLA}_{2}\right)$, secreted $\left(\mathrm{SPLA}_{2}\right)$, calciumindependent ( $\left.\mathrm{iPLA}_{2}\right)$, platelet-activating factor acetylhydrolase (PAF-AH) also referred to as lipoprotein-associated phospholipase $A_{2}$ (LP-PLA2), lysosomal phospholipase $A_{2}$ (L-PLA2), and adipose-PLA 2 (AdPLA) [1]. During the last decade, it has been shown that $\mathrm{PLA}_{2} \mathrm{~S}$ are associated with several inflammatory diseases including asthma, arthritis, and cancer, and thus constitute an attractive target for inhibitor development [6-8].

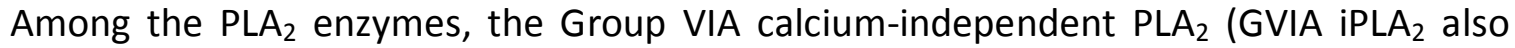
known as PNPLA9) has attracted considerable pharmaceutical interest because it is implicated in many diseases including diabetes [9], Barth syndrome [10], and NBIA/Neuroaxonal Dystrophy [11], and thus, the development of potent inhibitors that are also highly selective for GVIA iPLA 2 may lead to new therapeutics. The association of GVIA iPLA 2 with membranes and the lack of an X-ray crystal structure to assist in silico design render the developments of inhibitors very challenging. The human GVIA iPLA 2 gene was found to express multiple splice variants, with transcript variant 1 to be one of the two active isoforms in humans. Splice variant 1 contains 806 amino acids, and a molecular weight of $90 \mathrm{kDa}$. Based on its sequence, GVIA iPLA $A_{2}$ is composed of 7 ankyrin repeats, a linker region that contains an insert of 54 amino acids disrupting the eighth ankyrin repeat, and a patatin-like $\alpha / \beta$ hydrolase catalytic domain. The enzyme belongs to the carboxylesterase family that utilizes a catalytic dyad of Ser/Asp instead of the conventional carboxylesterase catalytic triad of Ser/His/Glu or Asp [1]. Even though there is no available crystal structure for GVIA iPLA 2 , molecular docking and dynamics (MD) simulations guided by hydrogen/deuterium exchange mass spectrometry (DXMS) data have successfully been used in the past by our group, to study the interactions of a GVIA iPLA 2 homology model with membranes, substrates and inhibitors [12-14].

Several compounds have been reported to inhibit the enzymatic activity of GVIA iPLA 2 including the $S$-enantiomer of the historically used covalent inhibitor bromoenol lactone (S-BEL) [15]. Although BEL potently inhibits the enzyme, it has low selectivity because it also inhibits other serine proteases. Methyl arachidonyl fluorophosphonates (MAFP) are also potent GVIA $\mathrm{iPLA}_{2}$ inhibitors, but they are irreversible, and not specific for iPLA ${ }_{2}$ [16]. Another class of GVIA $\mathrm{iPLA}_{2}$ inhibitors is the 2-oxoamides based on dipeptides and pseudo dipeptides, but they also inhibit CPLA 2 [17]. In 1995, fatty acyl trifluoromethylketones (TFKs) were identified as promising GVIA iPLA 2 inhibitors that target the catalytic Ser [15]. Further, development of various 
polyfluroketones by Kokotos et al led to a series of small aromatic compounds as potent and selective inhibitors of human GVIA iPLA $2[18,19]$.

TFKs are well known in mammalian pharmacology, and they were reported as transition state esterase inhibitors $[20,21]$. They were also found to be potent inhibitors of an insect juvenile hormone esterase [22]. Thioether TFK analogues were found to be 10-50 times more potent [23] and active in vivo to disrupt insect development [24]. However, these analogues were not extensively studied in mammalian enzymes compared to the non-thioether ones. The first mammalian enzyme that the beta-thio TFKs were found to be potent as inhibitors is carboxylesterase, which also exhibited an increased potency over the non-thio TFKs [25-28]. In spite of multiple studies and X-ray structures of the thio TFKs in esterase catalytic sites, the exact contribution of the thioether to inhibitor potency remains elusive [29]. The sulfur atom was initially added into TFKs as a bioisosteric replacement of the olefin group in juvenile hormone and it increased the efficacy of TFKs greater than expected. It is well known that the carbonyl group in TFKs exists in equilibrium with the hydrate (gem diol) form, because the polarization of the ketone is enhanced by the fluorine atoms [30,31]. One of the hypotheses related to the increased efficacy of the thio TFKs is that the sulfur atom shifts the equilibrium towards the gem diol form [32]. An alternative hypothesis is that the sulfur atom may participates in $\pi$-stacking interactions with aromatic residues in the enzyme active site [26].

In this study a series of beta-substituted TFKs containing a sulfur, oxygen and selenium atom at the beta-carbon atom were tested towards human Group VIA calcium-independent, Group IVA cytosolic and Group V secreted PLA2. TFKs were previously synthesized by Hammock's group and were initially tested toward juvenile hormone esterase and mammalian carboxylesterases $[26,27]$. Beta-thio TFKs exhibited inhibition against GVIA iPLA 2 while they don't show significant activity towards GIVA CPLA 2 and GV SPLA 2 . The most potent and selective inhibitor 3 (OTFP) exhibited an $X_{1}(50)$ of 0.0002 mole fraction corresponding to $\mathrm{IC}_{50}$ of $110 \mathrm{nM}$. In the absence of a crystal structure for GVIA iPLA 2 , DXMS was employed to identify the peptide regions of GVIA iPLA 2 showing decreased deuteration levels upon binding with the OTFP. The DXMS data were used to guide molecular docking and dynamics (MD) simulations in the presence of a POPC membrane patch. Clustering analysis led to an enzyme-inhibitor complex that revealed a detailed binding mode of OTFP in the enzyme binding site. This binding mode occupies $70 \%$ of the binding modes occurring during the MD simulation. The resulted 3D complex was used to dock all the compounds with $X_{1}(50)$ values and the established SAR model exhibited very good linearity between the theoretical binding score (XP GScore) and the experimental inhibitory activity $\left(r^{2}\right.$ of 0.8$)$. The docking complexes provide insight into the structure-activity relationship (SAR) that will be used to design, synthesize and test new betathioether analogues in order to identify compounds with improved inhibitory properties against GVIA iPLA . This work represents a novel multidisciplinary approach showing that the 
combination of computer-aided design along with experimental techniques is a powerful tool in drug discovery.

\section{Results and Discussion}

\subsection{Inhibition of human GVIA iPLA, GIVA cPLA 2 and GV SPLA}

Trifluoromethylketones (TFKs) were tested for their inhibitory activity against the human recombinant PLA $A_{2}$ enzymes using a mixed micelle modified Dole assay [33, 34]. Table 1 summarizes the structure and inhibitory activity of TFKs towards three human PLA $\mathrm{L}_{2}$ enzymes. Initial screening was performed for each PLA $\mathrm{P}_{2}$ enzyme at 0.091 mole fraction of the compound in the total surface volume of the mixed micelles, and then the $X_{1}(50)$ was determined for the compounds that exhibited more than $95 \%$ inhibition. An exception was made for compound 1 because it was used for the SAR. $X_{1}(50)$ is defined as the mole fraction of the compound in the total PAPC substrate/Triton X-100 mixed micelle surface required to inhibit $50 \%$ of the enzyme [33]. Mole fraction is a dimensionless number derived by dividing the number of moles of inhibitor by total number of moles of substrate plus inhibitor plus detergent. $X_{1}(50)$ values can be converted to molar concentration by considering that 0.091 mole faction of inhibitor corresponds to a $50 \mu \mathrm{M}$ concentration. Thus, an $X_{1}(50)$ of 0.0002 corresponds to $110 \mathrm{nM}$.

Among the TFKs containing an aliphatic chain (1-6, Table 1) compound 3 (OTFP), which contains eight carbon atoms in its aliphatic chain, exhibited the highest inhibition against GVIA $\mathrm{iPLA}_{2}$ (see sigmoidal response curve in Fig. S1), while it did not show significant inhibition

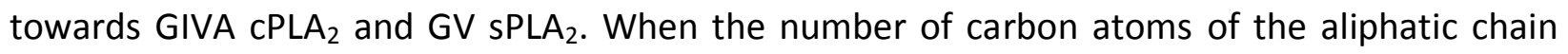
was decreased by two in compound 2 , the activity against GVIA iPLA 2 was also reduced by twofold. Further decrease of the number of carbon atoms by four in compound 1 reduced the inhibitory activity by two orders of magnitude. Increasing the carbon atoms of the aliphatic chain by two in compound 4 and three in compound 5 reduced the inhibitory activity against GVIA iPLA 2 by 1.5 and 2.5-fold, respectively, while the two compounds also lost selectivity since they also inhibited GIVA cPLA 2 , even though their inhibitory activity against GIVA CPLA 2 was one and two orders of magnitude, respectively, less in comparison with GVIA iPLA 2 . Further increases in the number of carbon atoms of the aliphatic chain by four in compound 6 reduced the inhibitory activity against GVIA iPLA 2 by one order of magnitude. According to the above results, the size of the aliphatic chain is crucial for the potency and selectivity of these inhibitors against GVIA iPLA 2 with the optimum number of carbon atoms being eight in compound 3 (OTFP). Thus, by optimizing the hydrophobic chain of beta-thio TFKs, it is possible to improve potency and selectivity toward GVIA iPLA 2 versus GIVA CPLA 2 since these properties depend on the size of the hydrophobic chain.

Compounds 7-17 contain an aromatic ring in the hydrophobic chain (Table 1). Compounds 7-15 contain a phenyl group directly connected to the sulfur atom and they exhibited similar inhibition against GVIA iPLA 2 , except for compound $\mathbf{1 3}$ which contains a polar 
nitro group on the phenyl ring. Slight differences in the inhibitor activity towards GVIA iPLA 2 depend on the position of the various substitutions on the phenyl ring, while none of them significantly inhibits GIVA cPLA 2 and GV sPLA 2 . Compound 16 (PETFP), which contains two extra carbon atoms between the sulfur atom and the phenyl ring exhibited the highest inhibitory activity among this set of compounds. It is worth mentioning that the inhibitory activity of PETFP is almost the same as the one exhibited by compound $\mathbf{2}$ and two-fold less than the inhibitory activity of OTFP. PETFP and $\mathbf{2}$ have similar sizes since the number of carbon atoms from the sulfur atom to the end of the chain is six. The above results support further the notion that by optimizing the hydrophobic chain of beta-thio TFKs, it is possible to alter potency and selectivity towards GVIA iPLA2. Finally, it seems that the sulfur atom plays an important role on the inhibitory activity of these compounds because compound $\mathbf{1 7}$ which contains a selenium atom showed insignificant activity. While the size of the hydrophobic chain plays a significant role in the selectivity of beta-thio TFKs, it is clear that the sulfur atom is vital for the potency of these analogues. Thus, altering the beta-thioether and the hydrophobic chain can be consider important tools in designing new more potent and selective analogues.

Compounds 18-26 are variously substituted-TFKs. Compound 18 that contains an oxygen atom exhibited an order of magnitude less inhibitory activity against GVIA iPLA 2 than its sulfur analogue $\mathbf{2}$ showing the importance of the sulfur atom for the potency of these compounds. Compound 19 showed very low activity towards GVIA iPLA 2 indicating the importance of the carbonyl group for the inhibitory activity of these compounds. Compounds 20-26 contain various substitutions of the sulfur atom and the carbonyl group and none of them exhibited significant inhibition against any of the three PLA 2 groups, further supporting the necessity of the sulfur atom and the carbonyl group for the inhibitory activity of TFKs.

Table 1. Inhibition of Human GVIA iPLA 2, GIVA cPLA $_{2}$, and GV sPLA 2 by $\mathrm{TFKs}^{\alpha}$

\begin{tabular}{|c|c|c|c|c|c|c|}
\hline \multirow{2}{*}{ No } & \multirow{2}{*}{ Structure } & \multicolumn{2}{|c|}{ GVIA iPLA $_{2}$} & \multicolumn{2}{|c|}{ GIVA CPLA $_{2}$} & \multirow{2}{*}{$\frac{\mathrm{GV} \mathrm{sPLA}_{2}}{\% \text { Inhibition }}$} \\
\hline & & \% Inhibition & $X_{1}(50)$ & \% Inhibition & $X_{1}(50)$ & \\
\hline 1 & $\mathrm{C}_{4} \mathrm{H}_{9}$ & $91 \pm 1$ & $0.07 \pm 0.04$ & $46 \pm 7$ & $\ldots$ & $14 \pm 12$ \\
\hline 2 & $\mathrm{C}_{6}$ & $99 \pm 1$ & $0.00052 \pm 0.00005$ & $77 \pm 3$ & $\ldots$ & $33 \pm 10$ \\
\hline 3 & $\mathrm{C}_{8} \mathrm{H}$ & $99 \pm 0.2$ & $0.00020 \pm 0.00002$ & $82 \pm 1$ & $\ldots$ & $68 \pm 3$ \\
\hline 4 & & $99 \pm 0.3$ & $0.00041 \pm 0.00003$ & $100 \pm 1$ & $0.008 \pm 0.001$ & $78 \pm 2$ \\
\hline 5 & & $98 \pm 0.2$ & $0.0007 \pm 0.0001$ & $98 \pm 0.2$ & $0.014 \pm 0.002$ & $73 \pm 0.4$ \\
\hline
\end{tabular}




\begin{tabular}{|c|c|c|c|c|c|c|}
\hline 6 & & $97 \pm 0.2$ & $0.0043 \pm 0.0009$ & $83 \pm 1$ & $\ldots$ & $87 \pm 5$ \\
\hline 7 & & $89 \pm 1$ & $\ldots$ & $75 \pm 5$ & $\ldots$ & $27 \pm 3$ \\
\hline 8 & & $95 \pm 1$ & $0.009 \pm 0.001$ & $61 \pm 2$ & $\ldots$ & $18 \pm 9$ \\
\hline 9 & & $93 \pm 1$ & $0.011 \pm 0.002$ & $70 \pm 1$ & $\ldots$ & $16 \pm 9$ \\
\hline 10 & & $46 \pm 5$ & $\ldots$ & $24 \pm 2$ & $\ldots$ & $14 \pm 8$ \\
\hline 11 & & $95 \pm 1$ & $0.011 \pm 0.002$ & $82 \pm 1$ & $\ldots$ & $23 \pm 3$ \\
\hline 12 & & $97 \pm 1$ & $0.0071 \pm 0.0007$ & $82 \pm 0.4$ & $\ldots$ & $35 \pm 4$ \\
\hline 13 & & $52 \pm 3$ & $\ldots$ & $26 \pm 4$ & $\ldots$ & $17 \pm 5$ \\
\hline 14 & & $98 \pm 1$ & $0.011 \pm 0.002$ & $91 \pm 1$ & $\ldots$ & $46 \pm 4$ \\
\hline 15 & & $99 \pm 1$ & $0.0049 \pm 0.0005$ & $91 \pm 1$ & $\ldots$ & $43 \pm 7$ \\
\hline 16 & & $100 \pm 4$ & $0.00059 \pm 0.00005$ & $92 \pm 1$ & $\ldots$ & $61 \pm 2$ \\
\hline 17 & & $46 \pm 12$ & $\ldots$ & $18 \pm 2$ & $\ldots$ & $44 \pm 3$ \\
\hline 18 & & $99 \pm 0.2$ & $0.006 \pm 0.001$ & $76 \pm 5$ & $\ldots$ & $35 \pm 5$ \\
\hline 19 & & $43 \pm 3$ & $\ldots$ & $58 \pm 9$ & $\ldots$ & $35 \pm 7$ \\
\hline 20 & & $39 \pm 8$ & $\ldots$ & $60 \pm 4$ & $\ldots$ & $66 \pm 4$ \\
\hline 21 & & $15 \pm 8$ & ... & $5 \pm 5$ & $\ldots$ & $16 \pm 9$ \\
\hline 22 & & $24 \pm 10$ & $\ldots$ & $5 \pm 5$ & $\ldots$ & $23 \pm 8$ \\
\hline 23 & & $32 \pm 7$ & $\ldots$ & $2 \pm 8$ & $\ldots$ & $80 \pm 4$ \\
\hline
\end{tabular}




\begin{tabular}{|c|c|c|c|c|c|c|}
\hline 24 & $\mathrm{C}_{6} \mathrm{H}_{13}-\mathrm{S}_{\mathrm{O}}^{\mathrm{O}}$ & $71 \pm 2$ & $\ldots$ & $14 \pm 4$ & $\cdots$ & $0 \pm 0$ \\
\hline 25 & & $82 \pm 1$ & $\ldots$ & $19 \pm 5$ & $\ldots$ & $18 \pm 8$ \\
\hline 26 & & $52 \pm 1$ & $\ldots$ & $34 \pm 5$ & $\ldots$ & $44 \pm 2$ \\
\hline
\end{tabular}

${ }^{\alpha}$ Average percent inhibition and standard error $(n=3)$ are reported for each compound at 0.091 mole fraction. $X_{1}(50)$ values were determined for inhibitors with greater than $95 \%$ inhibition, except for compound 1 which was included in the SAR.

\subsection{Defining the binding site of OTFP using DXMS}

Hydrogen/deuterium exchange mass spectrometry (DXMS) [35] is a powerful technique used to study the structural dynamics of membrane proteins [36, 37] as well as the association of PLA $A_{2}$ enzymes with membranes $[38,39]$. With DXMS we are able to capture the exchange rates of the backbone amide hydrogen atoms of an enzyme with deuterium atoms after incubation of the enzyme with deuterium dioxide. This method was successfully combined with computational methods to study the interactions of $\mathrm{PLA}_{2}$ enzymes with membranes, substrates and inhibitors $[12,14,40]$. The combination of DXMS experimental data with molecular docking and dynamics simulations has been proven very powerful in elucidating the binding mode of OTFP in the binding site of GVIA iPLA 2 considering that the X-ray crystal structure of the enzyme has not been solved yet.

\subsection{Digestion Map of GVIA iPLA}

The enzyme digestion was optimized to give a peptide map with the highest coverage of GVIA iPLA 2 sequence as previously described [14]. The optimized condition yielded 174 distinct peptides that gave $90 \%$ coverage of the GVIA iPLA 2 sequence (Fig. S2). Among these peptides, 70 peptides with the best signal-to-noise ratio were used for the analysis.

\subsection{Interactions of OTFP with GVIA iPLA 2 binding site}

According to the inhibition studies OTFP is a competitive reversible inhibitor (sigmoidal response curve, Fig. S1). As a result, deuteration changes upon OTFP binding were expected around the binding site of GVIA iPLA . In particular, seven peptide regions surrounding the binding site, residues 481-493 (yellow), 514-524 (magenta), 542-562 (orange), 630-655 (green), 656-664 (pink), 718-730 (red), and 770-778 (blue) in Fig. 1, exhibited the most significant decrease of deuteration levels (Table 2). None of these peptides showed an increase in deuteration after the binding experiment with OTFP. Region 481-493 in the absence of OTFP showed an average of $43 \%$ deuteration, while in the presence of OTFP showed a $27 \%$ deuteration which is a decrease of approximately $16 \%$. This region contains the oxyanion hole (Gly486/Gly487) that stabilizes the tetrahedral intermediate during the hydrolysis of a phospholipid substrate [12]. Region 542-562 contains the catalytic serine (Ser519), and showed 
an average decrease of $13 \%$ deuteration in the presence of OTFP. Region $630-655$ contains the catalytic aspartic acid (Asp652), and showed a decrease of approximately $12 \%$ deuteration in the presence of OTFP. Regions 656-664 and 718-730 are located near the catalytic aspartic acid (Asp652), and they showed an average decrease of $12 \%$ and $13 \%$ deuteration in the presence of OTFP, respectively. These regions contain residues like Asn658 and Lys725 that might interact with the fluorine atoms of OTFP. Peptide region 718-730 is part of the membrane anchor region 708-730 that was found to show more than $40 \%$ decrease in deuteration rates in the presence of PAPC vesicles [38]. Using coarse-grained and atomistic MD simulations, we have elucidated the association mechanism of this region with the membrane [13]. It is worth mentioning that in the presence of the OTFP inhibitor part of the same region (residues 718-730) showed only $13 \%$ decrease of deuteration level. Finally, regions 542-562 and 770-778 contain mostly hydrophobic residues that might interact with the alkyl chain of OTFP, and they both showed a significant decrease of deuteration level in the presence of the inhibitor $(18 \%$ and $20 \%$, respectively).

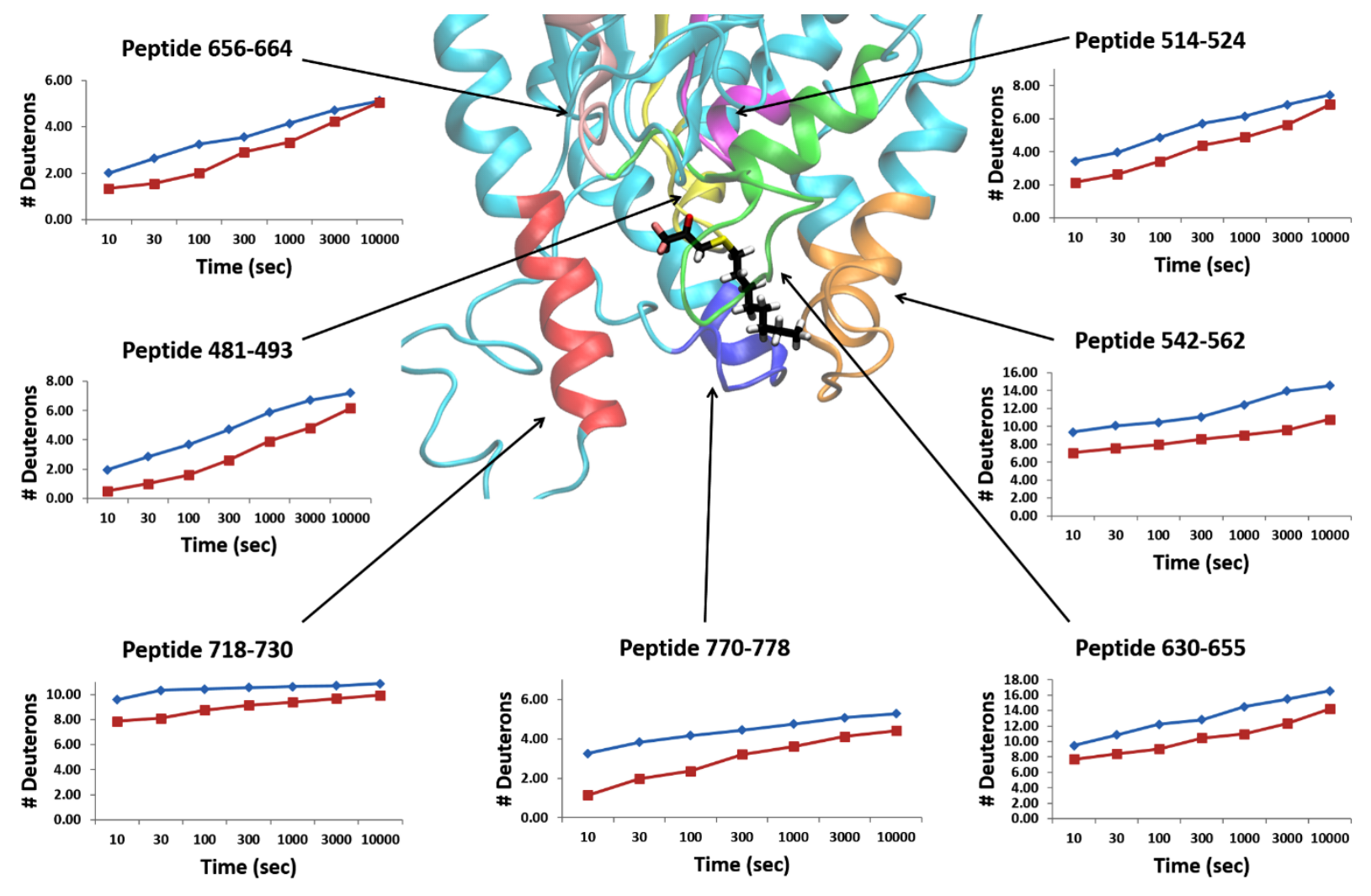

Fig. 1. Hydrogen/Deuterium exchange results upon binding of OTFP to GVIA iPLA 2 binding site. Percentage of deuteration level is presented for each peptide region of GVIA $\mathrm{PPLA}_{2}$ in the absence (blue) and presence (red) of OTFP. For each curve seven time points were studied and seven different regions showed a decrease of deuteration rates upon OTFP binding: residues 
481-493 (yellow), 514-524 (magenta), 542-562 (orange), 630-655 (green), 656-664 (pink), 718730 (red), and 770-778 (blue).

Table 2. Average percentage of deuteration level of the peptide regions that showed decreased exchange rates upon binding with OTFP.

\begin{tabular}{|c|c|c|c|}
\hline Peptide Region & $\begin{array}{c}\text { Average \% } \\
\text { Deuteration } \\
\text { (absence of OTFP) }\end{array}$ & $\begin{array}{c}\text { Average \% } \\
\text { Deuteration } \\
\text { (Presence of OTFP) }\end{array}$ & AAverage \% Deuteration \\
\hline 481-493 (yellow) & 43 & 27 & 16 \\
\hline $514-524$ (magenta) & 61 & 48 & 13 \\
\hline $542-562$ (orange) & 69 & 51 & 18 \\
\hline $630-655$ (green) & 60 & 48 & 12 \\
\hline $656-664$ (pink) & 61 & 49 & 13 \\
\hline $718-730$ (red) & 95 & 82 & 20 \\
\hline $770-778$ (blue) & 63 & 43 & \\
\hline
\end{tabular}

\subsection{Binding mode of OTFP in GVIA iPLA 2 binding site}

The binding mode of OTFP was calculated computationally using the homology model of GVIA iPLA 2 [12] and the Induced Fit Docking (IFD) protocol implemented in Schrödinger suite [41]. Residues Gly486, Gly487, Lys489, Ser519, Val548, Phe549, Leu560, Tyr643, Phe644, Asp652, Lys729, and Leu770 were selected to define the binding site of GVIA IPLA 2 in the IFD calculations. These residues exhibited significant decreased percentage of deuteration levels in DXMS studies upon binding of OTFP inhibitor (see DXMS results). According to the 3D GVIA iPLA $A_{2}$-OTFP complex generated by IFD (Fig. 2A) the trifluoromethylketone group is located in the hydrophilic region of GVIA iPLA 2 binding site with the oxygen atom of the carbonyl group participating in one hydrogen bond ( $\mathrm{H}$-bond) with the catalytic Ser519. The fluorine atoms are in spatial proximity with residues Asn658 and Lys725. The alkyl chain of the inhibitor is accommodated near the entrance of the binding site interacting with residues such as Val548, Phe549, Leu560, Tyr643, Phe644, and Leu770. 

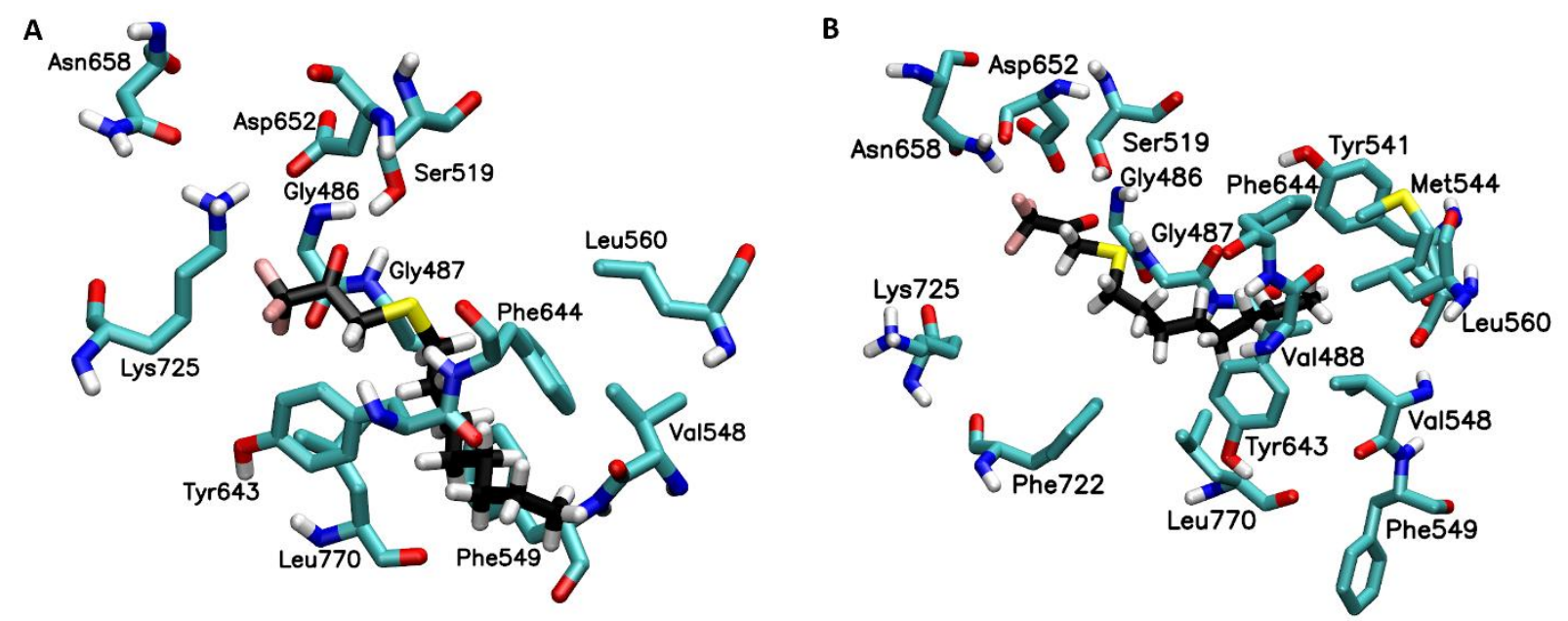

Fig. 2. Binding mode of OTFP in the GVIA iPLA 2 binding site. (A) Initial complex generated by IFD. (B) Complex after the MD simulation.

The GVIA iPLA 2 -OTFP IFD complex was placed on the surface of a POPC membrane patch (Fig. 3), solvated with explicit water molecules, minimized, equilibrated and subjected to a 300 ns simulation using NAMD 2.9 [42]. The structural stability of the complex was indicated by a stable root mean square deviation (RMSD) of the backbone atoms aligned to the GVIA PPLA OTFP IFD complex, which was stabilized at $\sim 2.3 \AA$ showing that the simulation was reasonably converged (Fig. S3). Figure 2B and Movie 1 shows the binding mode of OTFP after the MD simulation. In the hydrophilic area of the binding site the oxygen atom of the carbonyl group participates in H-bonding with residues Gly486/Gly487 the so called "oxyanion hole". The distances between $\mathrm{H}$-bond acceptor and donor are $\sim 3 \AA$ after the first $150 \mathrm{~ns}$ of the simulation and until the end (Fig. S4A). Movie 2 shows the interactions of the fluoroketone group occur during the simulation. The catalytic Ser519 does not form any $\mathrm{H}$-bonds with the carbonyl group even if they are in spatial proximity ( $\sim 3 \AA$ after the first 150 , Fig. S4A), because the hydrogen atom of the hydroxyl group doesn't not adopt a suitable orientation to form an H-bond (Movie 2). Ser519 constantly forms H-bonding with the catalytic Asp652 (Movie 2). It has been argued for years that TFKs exist in equilibrium between the ketone and the hydrate form. It is possible that fluorine and sulfur atoms shift the equilibrium in favor of the hydrate form, however, the ketone is considered to be the reactive form of the inhibitor, which might undergoes nucleophilic attack by the hydroxyl group of catalytic serine (Ser519-OH) [27]. During the simulations the oxygen atom of the carbonyl group is constantly interacting with the "oxyanion hole" (Gly486/Gly487), while the carbon atom of the carbonyl group is very close to catalytic Ser519 suggesting that its hydroxyl group is in spatial proximity to form a reversible hemiketal (Movie 2). It is likely that the hemiketal is stabilized by the "oxyanion hole" (Gly486/Gly487), however, no one has been able to definitively show that the carbonyl group forms a hemiketal with the catalytic serine. The fluorine atoms interact with Asn658 ( 3.3 $\AA$, Fig. S4A) but no interactions were observed with Lys725 (Movie 2). 


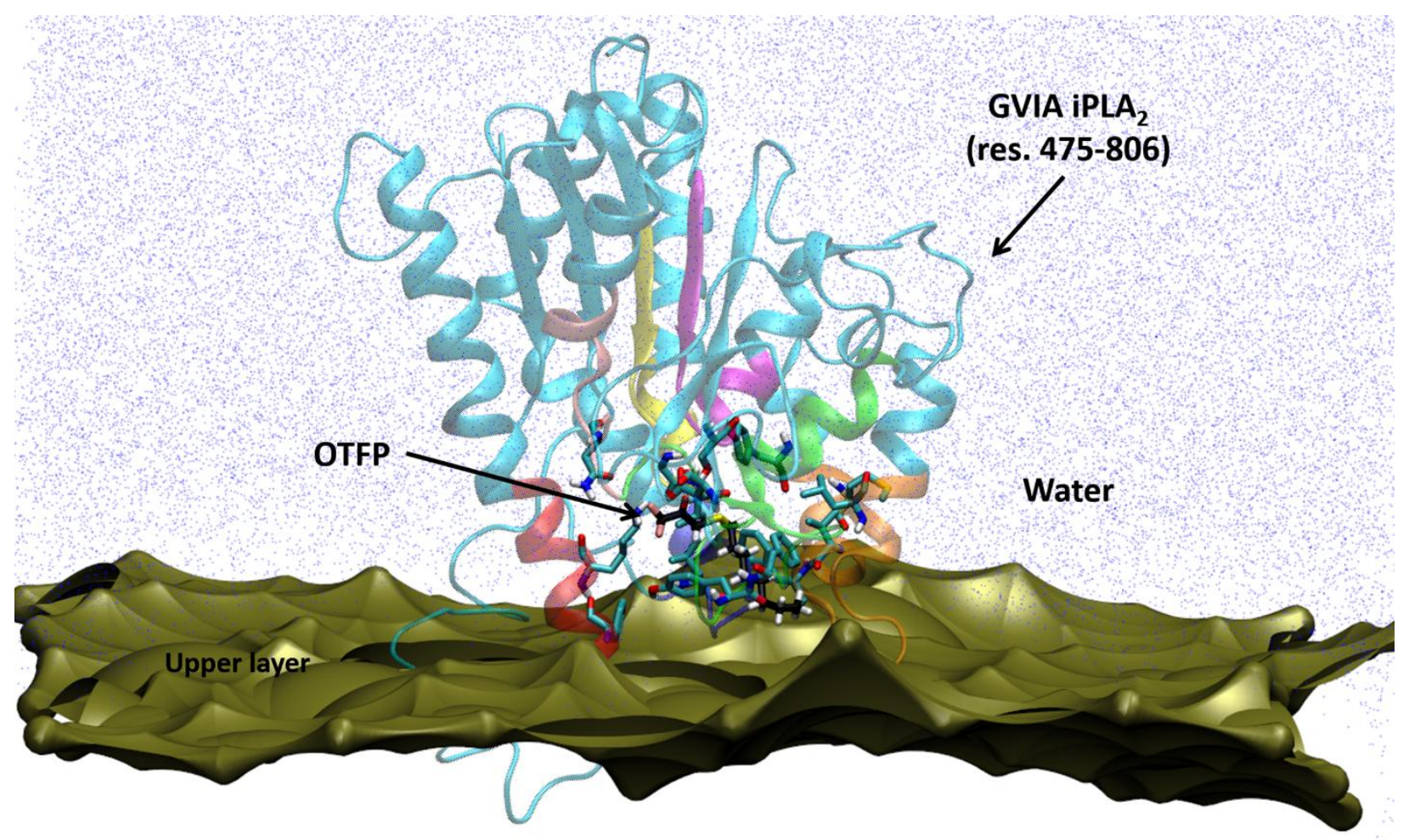

POPC membrane

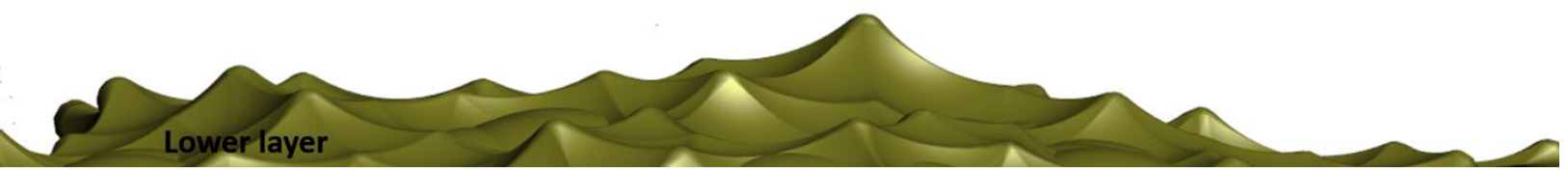

Figure 3. GVIA iPLA 2 -OTFP IFD complex on the surface of a POPC membrane patch and solvated with water molecules.

The alkyl chain moves from the entrance into the hydrophobic region of the binding site (Movie 1). Clustering analysis revealed that $70 \%$ of the snapshots saved in the MD trajectory show the hydrophobic chain of OTFP in a horizontal (Fig. 2B and Movie 1), rather than a vertical orientation suggested by IFD (Fig. 2A). The simulation showed the hydrophobic chain to interact with residues like Val488, Ile523, Tyr541, Met544, Val548, Phe549, Leu560, Tyr643, Phe644 and Leu770 (Movie 1). Our previously published work showed that the same hydrophobic region is occupied by the sn-2 fatty acyl chain of a PAPC substrate molecule [12]. The sulfur atom is in spatial proximity with Gly487 ( 3.9 $\AA$ acceptor-donor distance, Fig. S4B) which is part of the "oxyanion hole" suggesting the formation of a weak H-bond (Movie 2). Two aromatic residues, Tyr643 and Phe722 are also located near the sulfur atom competing for pi-pi stacking interactions (Movie 3). The distance of the phenyl ring centroid of Tyr643 is $\sim 4$ and is increasing to $\sim 5 \AA$ when the phenyl ring of Phe722 is approaching the sulfur atom (Fig. S4B). Sulfur is a highly versatile atom in terms of the molecular interactions that it can form in 
molecular recognition. It has the ability to interact with electron-poor and with electron-rich functional groups [43]. The backbone amide hydrogen atom of Gly487 is electron-poor, while the aromatic rings of Tyr643 and Phe722 are electro-rich because of their $\pi$ electron cloud and it is possible that the sulfur atom is involved in interactions with these residues (Movie 2 and 3). This observation is in agreement with the hypothesis suggested by Wheelock and his coworkers that the sulfur atom enhances the efficacy of TFKs because is involved in $\pi$-stacking interactions with aromatic residues of the active site [26].

\subsection{Structure-activity relationship (SAR)}

Five representative enzyme-inhibitor complexes were chosen from the five clusters resulted from the clustering analysis for docking calculations. These complexes were optimized using the Protein Preparation Wizard (PPW) [44] and the OTFP inhibitor was redocked into the GVIA iPLA2 binding site using Glide [45]. The complex that received the highest XP GScore (Extra-Precision Glide Score) and was used to perform docking calculations on the set of 14 inhibitors for which $X_{1}(50)$ values were measured. The theoretical binding scores (Table 3 ) were correlated with $-\log X_{1}(50)$ values and an $r^{2}=0.8$ suggested very good correlation between theoretical and experimental results (Fig. 4).

Table 3. TFKs employed to generate SAR.

\begin{tabular}{|c|c|c|c|c|}
\hline No & Structure & $-\log X_{1}(50)$ & $\begin{array}{c}\text { XP GScore } \\
\text { (GVIA iPLA })\end{array}$ & $\begin{array}{c}\text { XP GScore } \\
\text { (GIVA cPLA })\end{array}$ \\
\hline 3 & & 3.7 & -7.9 & -4.9 \\
\hline 4 & & 3.4 & -7.6 & -6.0 \\
\hline 2 & & 3.3 & -7.0 & $\ldots$ \\
\hline 16 & & 3.2 & -6.6 & $\ldots$ \\
\hline 5 & & 3.2 & -6.9 & -5.9 \\
\hline 6 & & 2.4 & -7.1 & $\ldots$ \\
\hline 15 & & 2.3 & -6.2 & ... \\
\hline 18 & & 2.2 & -6.6 & $\ldots$ \\
\hline
\end{tabular}




\begin{tabular}{|c|c|c|c|c|}
\hline 12 & 2.2 & -6.0 & $\ldots$ \\
\hline 8 & 2.1 & -5.9 & $\ldots$ \\
\hline 11 & 2.0 & -6.1 & $\ldots$ \\
\hline 14 & & 2.0 & -5.9 & $\ldots$ \\
\hline 1 & $\mathrm{C}_{4}$ & 1.2 & -5.7 & $\ldots$ \\
\hline
\end{tabular}

The SAR model suggests that docking calculations can be used to predict the potency of these compounds towards GVIA iPLA2. It should be noted that compounds with high $X_{1}(50)$ values tend to have higher XP GScore, while compounds with low $X_{1}(50)$ values tend to have a lower XP GScore within the acceptable error that is typical for docking calculations. In addition to the predictions of the binding score, the docking 3D complexes provide insight into the binding mode of these compounds. According to the docking complex, OTFP has the suitable size to fit into the binding site of GVIA iPLA 2 and optimize its binding exhibiting the higher inhibition against the enzyme (Fig. 4 and Table 3). PETFP and 2 lack interactions with the hydrophobic pocket of GVIA iPLA 2 due to shorter hydrophobic chains therefore their inhibition against the enzyme is 30-fold less than OTFP. The aliphatic chains of $\mathbf{2}$ and $\mathbf{1 8}$ are identical showing similar interactions with the hydrophobic region of the enzyme binding site. However, inhibitor 18 exhibited 12-fold less inhibition than inhibitor 2 due to replacement of the sulfur atom by an oxygen atom. The oxygen atom is smaller and its electron cloud is not as extended as the one of the sulfur atom resulting in weaker interactions of the oxygen atom with the aromatic residues of the binding site. The non-thio derivative of PETFP was previously tested and published as a GVIA iPLA 2 inhibitor with an $X_{1}(50)$ of 0.0096 [46]. PETFP exhibited 16-fold higher inhibition than its non-thio derivative. These two examples clearly indicate the significant role of the sulfur atom in improving the inhibitory potency of these compounds. Finally, the lower inhibition of $\mathbf{4}$ and $\mathbf{5}$ in comparison with OTFP indicating that eight is the optimum number of carbon atoms for the hydrophobic chain. Increases in the hydrophobic chain length results in weaker binding, probably due to steric clashes with the residues of the hydrophobic region in the enzyme binding site. 

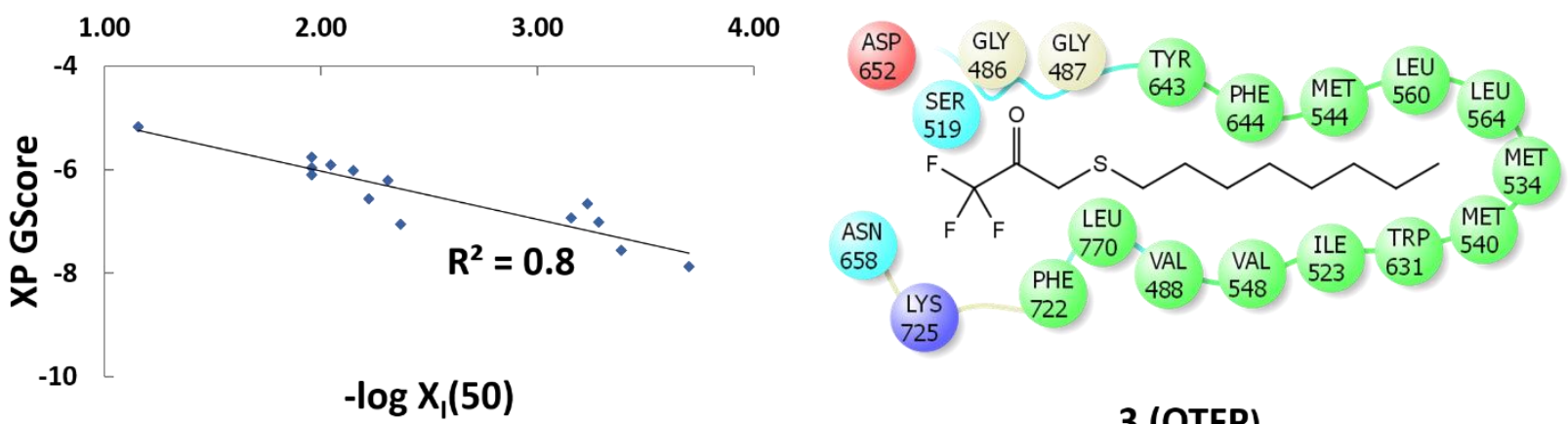

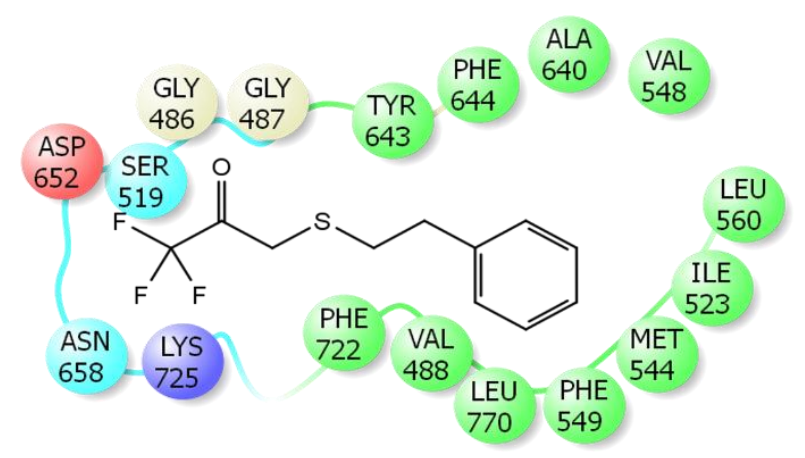

16 (PETFP)

\section{3 (OTFP)}

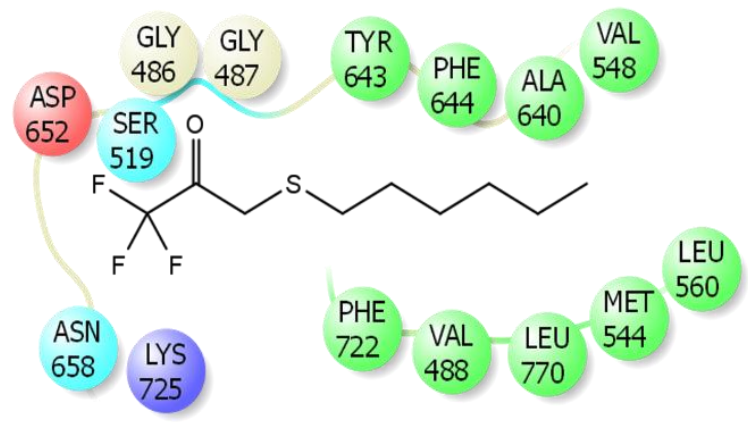

2

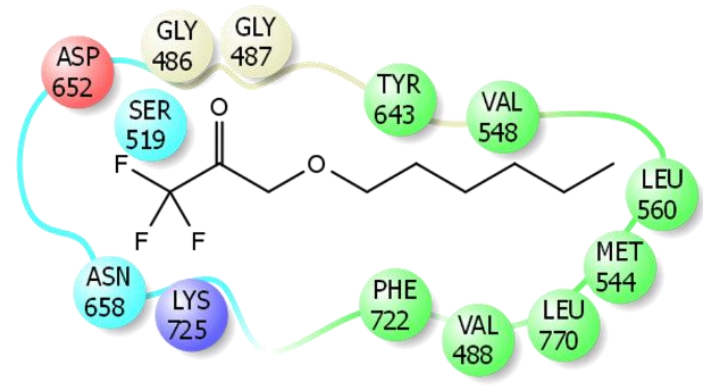

18

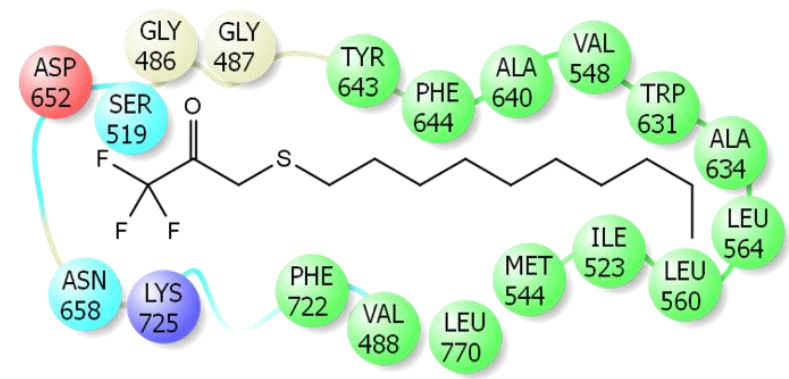

4

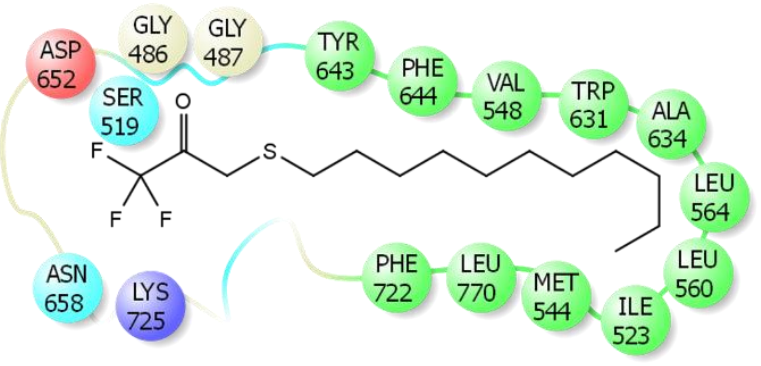

5

Figure 4. Correlation between theoretical XP GScore vs experimental -log $X_{1}(50)$ values and schematic representation of the interactions of representative inhibitors with GVIA iPLA 2 binding site. 
The docking calculation also revealed important information for the selectivity of these compounds. Inhibitor OTFP, 4, and 5 were docked in the binding site of GIVA CPLA 2 (Table 3 and Fig. 5). OTFP received lower XP GScore than inhibitors $\mathbf{4}$ and $\mathbf{5}$ but most importantly the docking complexes showed that the deep channel-like binding site of GVIA CPLA 2 requires longer hydrophobic chains for optimum binding of the inhibitor with ten being the optimum number of carbon atoms in inhibitor $\mathbf{4}$. The hydrophobic chain of inhibitor $\mathbf{4}$ and $\mathbf{5}$ is accommodated in GIVA CPLA $_{2}$ more favorably in comparison with OTFP showing interactions with additional residues of the hydrophobic region including Leu298, lle304 and Met390. The TFK group shows similar binding in the GVIA iPLA 2 binding site including $\mathrm{H}$-bonding of the oxygen atom with the "oxyanion hole" (Gly197/Gly198) and interactions of the fluorine atoms with Asn555 and Arg200. This indicated that the potency and selectivity of these compounds mostly depends on the binding of the sulfur atom and hydrophobic chain. Further optimization of the hydrophobic chain will lead to new analogues with improved potency and selectivity in terms of inhibition of GVIA iPLA 2 .
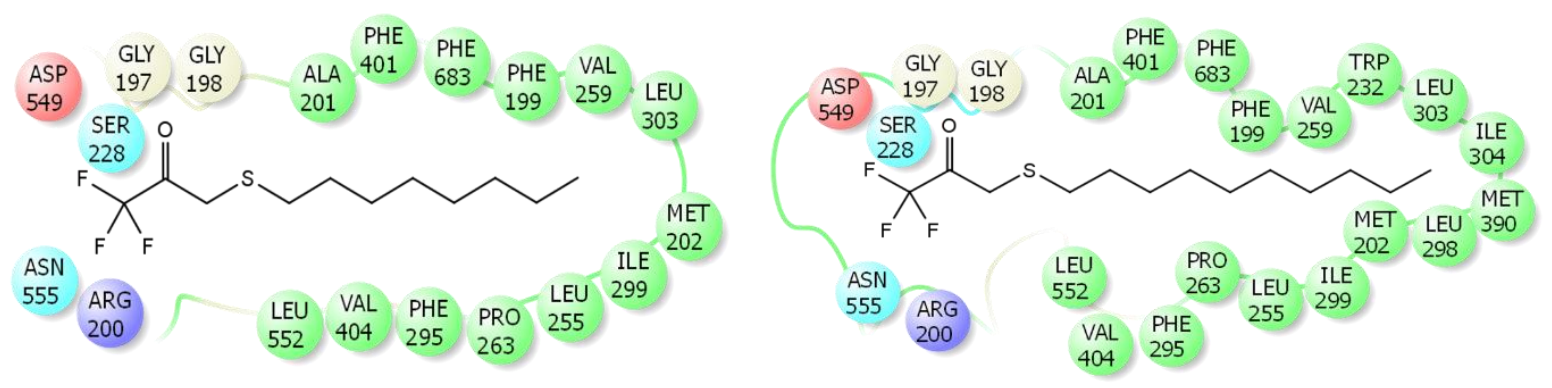

3 (OTFP)

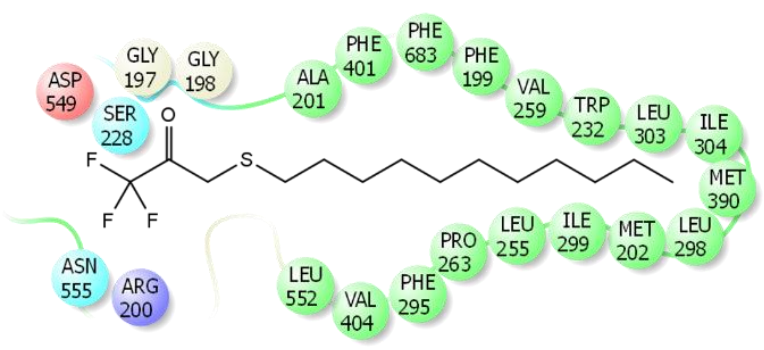

5

Figure 5. Schematic representation of the interactions of OTFP, 4 and 5 with GIVA CPLA 2 binding site.

\section{Conclusion}

In this study, beta-thio TFKs containing a hydrophobic chain were identified as potent and selective inhibitors for GVIA iPLA 2 . Compound OTFP which contains an eight carbon atom alkyl chain exhibited the highest inhibitory activity against the enzyme. This study also showed 
that the enzyme exhibits a preference for the size of the compound. By combining DXMS with computer-aided design techniques, the binding mode of the OTFP inhibitor in the binding site of the enzyme was identified. This is very important since no crystal structure has been solved for the enzyme co-crystallized with inhibitors to assist virtual screening for identifying new potent and selective inhibitors for GVIA iPLA 2 . Docking calculations led to the development of structure-activity relationship (SAR) conclusions and gave insight into the binding of these inhibitors. The docking complexes also revealed a detailed binding mode for these inhibitors suggestion that the potency and selectivity can be improved by optimizing the interactions of the hydrophobic chain. In future work, we will employ the SAR to discover new inhibitors for GVIA iPLA 2 as well as to further understand why the enzyme has a preference for small compounds, while its natural substrate consists of relatively large phospholipid molecules. This study will serve as a precursor for developing new potent and selective inhibitors not only for GVIA iPLA 2 , but also for GIVA CPLA 2 as well by altering the size and substitutions of the hydrophobic chain. New inhibitors for these two major cellular enzymes will serve as tools to further understand their mechanism of action as well as their biological functions and pathways.

\section{Experimental Section}

\subsection{Enzymatic Assays}

\subsubsection{Materials}

1-Palmitoyl-2-arachidonoyl-sn-glycero-3-phosphocholine (PAPC) was purchased from Avanti Polar Lipids, Inc. (Alabaster, Alabama 35007-9105). 1-palmitoyl-2-arachidonoyl-sn-glycero-3phosphocholine (PAPC), [arachidonyl- $1-{ }^{14} \mathrm{C}$ ]-, $10 \mu \mathrm{Ci}$ was purchased from PerkinElmer (Akron, $\mathrm{OH}$ 44311). Chemicals were purchased from Sigma-Aldrich (St. Louis, MO 63103) and all solvents are from Fisher Scientific (Pittsburgh, PA 15275) unless otherwise stated. Sf9 (Spodoptera frugiperda) insect cells and culture medium SF900 III SFM were purchased from Life Technologies (Grand Island, NY 14072 USA). Ni-NTA Agarose was obtained from QIAGEN (Valencia, CA 91355). Econo-Column chromatography columns and Bradford assay kits were obtained from Bio-Rad Laboratories (Hercules, CA 94547).

\subsubsection{Trifluoromethylketone (TFK) compounds}

The compounds were previously synthesized and purified as described $[26,27]$.

\subsubsection{Expression and purifications of $P L A_{2} S$}

The human GVIA iPLA 2 and GIVA CPLA $_{2}$ were expressed using the Baculovirus Expression Vector System (BEVS) and Sf9 cells as described elsewhere [39]. Sf9 cells were seeded in $1 \mathrm{~L}$ shaker flasks containing a suspension culture of $500 \mathrm{~mL}$ SF900 III SFM medium, and incubated at $27{ }^{\circ} \mathrm{C}$ for $24 \mathrm{~h}$ before infection. The cells were infected at density of $\sim 1.5 \times 10^{6}$ cells $/ \mathrm{mL}$ with a BEVS containing the cDNA of the N-terminal 6xHis-tag GVIA iPLA ${ }_{2}$ or C-terminal 6xHis-tag GIVA cPLA 2 at an MOI of 0.1 for 96 h. The cells were harvested by centrifugation ( $2000 \mathrm{rpm}, 5$ 
min, and $4{ }^{\circ} \mathrm{C}$ ), washed with PBS buffer containing protease inhibitors, and frozen at $-80{ }^{\circ} \mathrm{C}$ until purification. For purifying GVIA iPLA 2 , the cell pellet was resuspended in lysis buffer $(25 \mathrm{mM}$ Tris- $\mathrm{HCl}, 100 \mathrm{mM} \mathrm{NaCl}, 1 \mathrm{M}$ Urea, $2 \mathrm{mM}$ ATP, $10 \mathrm{mM}$ B-Mercaptoethanol, and protease inhibitors). After a mild sonication (10 seconds "on"/60 seconds "off" repeated for 3 times), the insoluble portion was removed by centrifugation at 13,000 rpm for $1 \mathrm{~h}$. The clear lysate was mixed with Ni-NTA resin, which was previously equilibrated in lysis buffer (1 mL Ni-NTA agarose was gently mixed with $4 \mathrm{~mL}$ lysis buffer, centrifuged at $1000 \mathrm{rpm}$ for $1 \mathrm{~min}$, and the supernatant was removed for 3 times), and mixed gently on a rotary shaker at $4{ }^{\circ} \mathrm{C}$ for $2 \mathrm{~h}$.

The lysate-Ni-NTA mixture was loaded on a column and washed with the wash buffer (25 mM Tris- $\mathrm{HCl}, 250 \mathrm{mM} \mathrm{NaCl}, 2 \mathrm{mM}$ ATP, and $10 \mathrm{mM}$ imidazole). Finally the enzyme was eluted with the elution buffer $(25 \mathrm{mM}$ Tris- $\mathrm{HCl}, 50 \mathrm{mM} \mathrm{NaCl}, 2 \mathrm{mM}$ ATP, and $250 \mathrm{mM}$ imidazole, and $30 \%$ glycerol), and dithiothreitol (DTT) was added to each elution fragment at a final concentration of $5 \mathrm{mM}$. The concentration of the enzyme was measured using the Bradford assay, and the purified enzyme was stored at $-80^{\circ} \mathrm{C}$. GIVA CPLA 2 was purified using the same buffers but without ATP. The final concentration of DTT in the elution fragment was 2 $\mathrm{mM}$, and the enzyme was stored at $-20^{\circ} \mathrm{C}$. The human $\mathrm{GV} \mathrm{sPLA} \mathrm{A}_{2}$ was expressed and purified as previously described by our lab and stored at $4{ }^{\circ} \mathrm{C}$ [47].

\subsubsection{PLA 2 activity assays}

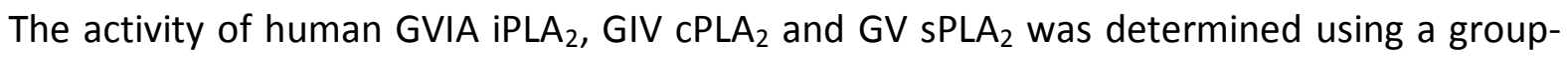
specific mixed micelle modified Dole assay [33,34]. The substrate was prepared using slightly different conditions for each enzyme to achieve optimum activity: (i) GVIA iPLA 2 mixed micelle substrate consisted of $400 \mu \mathrm{M}$ Triton $\mathrm{X}-100,98.3 \mu \mathrm{M}$ PAPC, and 1.7 $\mu \mathrm{M}$ arachidonyl-1 $-{ }^{14} \mathrm{C}$ PAPC in a buffer containing $100 \mathrm{mM}$ HEPES pH 7.5, 2 mM ATP, and 4 mM DTT; (ii) GIVA cPLA mixed $_{2}$ micelle substrate consisted of $400 \mu \mathrm{M}$ Triton X-100, 95.3 $\mu \mathrm{M}$ PAPC, $1.7 \mu \mathrm{M}$ arachidonyl-1 $-{ }^{14} \mathrm{C}$ PAPC, and $3 \mu \mathrm{M} \mathrm{PI}(4,5) \mathrm{P} 2$ in a buffer containing $100 \mathrm{mM}$ HEPES pH 7.5, $90 \mu \mathrm{M} \mathrm{CaCl2,} 2 \mathrm{mM}$ $\mathrm{DTT}$, and $0.1 \mathrm{mg} / \mathrm{ml} \mathrm{BSA}$; and (iii) GV sPLA 2 mixed micelles substrate consisted of $400 \mu \mathrm{M}$ Triton $\mathrm{X}-100,98.3 \mu \mathrm{M}$ PAPC, and $1.7 \mu \mathrm{M}$ arachidonyl- $1-{ }^{14} \mathrm{C}$ PAPC in a buffer containing $50 \mathrm{mM}$ Tris- $\mathrm{HCl}$ $\mathrm{pH} \mathrm{8.0,} \mathrm{and} 5 \mathrm{mM} \mathrm{CaCl} 2$. The compounds were initially screened at 0.091 mole fraction $(5 \mu \mathrm{L}$ of $5 \mathrm{mM}$ inhibitor in DMSO) in substrate (495 uL). $\mathrm{X}_{1}(50)$ was determined for compounds exhibiting greater than $93 \%$ inhibition. Inhibition curves were generated using GraphPad Prism 5.0 and the non-linear regression by plotting percentage of inhibition vs log (mole fraction) to calculate the reported $X_{1}(50)$ and its associated error.

\subsection{Hydrogen/Deuterium Exchange Mass Spectrometry (DXMS)}

\subsubsection{Preparation of deuterated samples.}

For preparing the deuterated samples, deuterium dioxide $\left(D_{2} \mathrm{O}\right)$ buffer containing $8,3 \mathrm{mM}$ Tris- $\mathrm{HCl}\left(\mathrm{pD}\right.$ of 7.5), $100 \mathrm{mM} \mathrm{NaCl}$ in $98 \% \mathrm{D}_{2} \mathrm{O}$ was used. DXMS experiments were performed according to the previously published method [14, 40]. Briefly, $20 \mu \mathrm{L}$ of GVIA iPLA (total $40 \mu \mathrm{g}$ of enzyme) were mixed with $60 \mu \mathrm{L}$ of $\mathrm{D}_{2} \mathrm{O}$ buffer to a final concentration of $73 \% \mathrm{D}_{2} \mathrm{O}$. Inhibitor 
binding experiments were performed incubating first GVIA iPLA 2 with $40 \mu \mathrm{M}$ of OTFP or DMSO for the control. The final concentration of DMSO was less than $1 \%$ for all experiments. The inhibitor was incubated with the enzyme for $30 \mathrm{~min}$ at room temperature. $\mathrm{D}_{2} \mathrm{O}$ buffer was added to the samples, and they were incubated for an additional 10, 30, 100, 300, 1000, 3000, and $10000 \mathrm{sec}$. The final concentration of OTFP, after the addition of $\mathrm{D}_{2} \mathrm{O}$ buffer was $10 \mu \mathrm{M}$. The deuterium exchange was quenched by adding $120 \mu \mathrm{L}$ of ice-cold quench solution containing $0.8 \%$ formic acid, $2 \mathrm{M}$ guanidine hydrochloride $(\mathrm{GdHCl})$, and $16.6 \%$ glycerol that acidified the samples to a final $\mathrm{pH}$ of 2.5 . The samples were immediately frozen on dry ice, and were stored at $-80{ }^{\circ} \mathrm{C}$ until analysis (usually within $48 \mathrm{~h}$ ).

\subsubsection{Proteolysis, Liquid Chromatography, and Mass Spectrometry Analysis.}

For proteolysis and mass spectrometry analysis, the samples were thawed and injected to a pepsin column for digestion as previously described [14, 40]. The proteolytic fragments (peptides) were separated on a C18 reversed phase analytical column at $0{ }^{\circ} \mathrm{C}$. The peptides were analyzed on an OrbiTrap Elite Mass Spectrometer (ThermoFisher Scientific, San Jose, CA). Peptides were analyzed with tandem mass spectrometry (MS/MS) data using SEQUEST (Thermo Finnigan, Inc.). The DXMS Explorer (Sierra Analytics Inc., Modesto CA) was employed to analyze the results and calculate the average of the mass envelope as previously described $[14,38]$.

\subsection{Computer-Aided Drug Design}

\subsubsection{Induced Fit Docking (IFD) protocol}

The GVIA iPLA 2 -OTFP initial complexes were generated using the IFD protocol implemented in Schrödinger Suite $[41,48]$. The 3D structure of GVIA iPLA (receptor) was prepared and optimized using the Protein Preparation Wizard (PPW) module [44]. Residues Gly486, Gly487, Lys489, Ser519, Val548, Phe549, Leu560, Tyr643, Phe644, Asp652, Lys729, and Leu770 were selected to define the binding site of GVIA iPLA 2 in the IFD calculations. The inhibitor 3D structures (ligand) were sketched using Maestro 9.9 sketcher, [49] and they were optimized using the LigPrep 3.2 application [50]. IFD protocol employs Glide 6.5 [45] and the refinement module in Prime 3.8 [51] to accurately predict enzyme-inhibitor complexes by incorporating side-chain flexibility for the receptor. The box center for the docking calculations was defined using the centroid of selected residues that were found to constitute the binding site of GVIA iPLA 2 using DXMS and extensive MD simulations in our previously published papers [12-14]. The box size was determined automatically according to the centroid of the specified binding site residues. For the initial docking of the inhibitors the side-chain of the binding site residues were trimmed automatically based on the B-factor. The receptor van der Waals scaling was set to 0.70 and the ligand van der Waals scaling to 0.50 . Twenty poses (binding modes) of each inhibitor were allowed to pass to the Prime refinement step. During the Prime refinement step, the side-chains of the residues within $6 \AA$ the inhibitor pose were optimized in terms of conformation and potential energy. Finally, twenty enzyme-inhibitor structures were allowed to pass to the redocking step with a threshold for eliminating high-energy structures from the 
Prime refinement of $30 \mathrm{kcal} / \mathrm{mol}$, and the Glide Extra-Precision (XP) scoring function [52]. The final complexes were selected based on the binding mode and the score.

\subsubsection{Molecular dynamics (MD) simulations}

MD simulations were carried on the IFD GVIA iPLA 2 -OTFP complex. The complex was placed on a POPC membrane patch of area $100 \times 100 \AA^{2}$ according to our previously published model [13]. POPC molecules within $0.6 \AA$ of the enzyme were removed, the system was solvated with TIP3P water molecules and sodium chloride at a concentration of $100 \mathrm{mM}$. The total number of atoms of the entire system was approximately 105,000. NAMD 2.9 was employed for the MD simulations. The CHARMM General Force Field (CGenFF) and parameters were used for the OTFP inhibitor, [53] and the CHARMM36 all-atom additive force field and parameters were used for the enzyme and the POPC membrane patch [54]. The system was minimized for 20,000 steps and equilibrated for 12 ns to relieve all the unfavorable contacts. Finally, a 300 ns MD simulation was performed at a temperature of $310 \mathrm{~K}$ using the NPT ensemble.

\subsubsection{Docking Calculations}

Clustering analysis was performed on the trajectories derived from the MD simulations on the enzyme-OTFP complex in order to identify suitable conformations for the docking calculations. The 3D structures were optimized using the PPW [44]. The 3D structures of the inhibitors were sketched using Maestro 9.9 sketcher [49] and they were optimized using LigPrep 3.2 [50]. Glide 5.8 was used for the rigid-docking of the compounds into the enzyme binding pocket [45]. The grid required for the docking procedure was generated using a scaling factor of 1.0 and partial charge cutoff of 0.25 , while $X, Y, Z$ dimensions of the inner box were set to $12 \AA$ around the docked inhibitor. For the inhibitor docking a scaling factor of 0.8 and partial charge cutoff of 0.15 were used that allow complete flexibility of the structures. The poses were selected according to the binding mode and the XP GScore. The Glide Extra-Precision (XP) scoring function was used for the calculations [52].

Acknowledgements. This work was supported by NIH Grant GM20501-40 (E. A. D) and by NIH, NSF, NBCR and HHMI (J.A.M). Partial support for this work was received from NIEHS R01 ES002710 and NIEHS Superfund Program P42 ES04699 (B. D. H.). This work used the Extreme

Science and Engineering Discovery Environment (XSEDE), which is supported by National Science Foundation grant number ACl-1053575. 


\section{References}

[1] E. Dennis, J. Cao, Y.-H. Hsu, V. Magrioti, G. Kokotos, Phospholipase $A_{2}$ enzymes: physical structure, biological function, disease implication, chemical inhibition, and therapeutic intervention, Chem. Rev., 111 (2011) 6130-6185.

[2] V.D. Mouchlis, E.A. Dennis, Membrane and inhibitor interactions of intracellular phospholipases $A_{2}$, Adv. Biol. Regul., (2015).

[3] M. Buczynski, D. Dumlao, E. Dennis, Thematic review series: proteomics. an integrated omics analysis of eicosanoid biology, J. Lipid Res., 50 (2009) 1015-1038.

[4] P.M. O'Byrne, Leukotrienes in the pathogenesis of asthma, Chest, 111 (1997) 27S-34S.

[5] M.E. Venable, S.C. Olson, M.L. Nieto, R.L. Wykle, Enzymatic studies of lyso platelet-activating factor acylation in human neutrophils and changes upon stimulation, J. Biol. Chem., 268 (1993) 7965-7975.

[6] E. Pniewska, R.C. Pawliczak, The involvement of phospholipases $A_{2}$ in asthma and chronic obstructive pulmonary disease, Mediators Inflamm., 2013 (2013) 793505.

[7] S. Masuda, M. Murakami, K. Komiyama, M. Ishihara, Y. Ishikawa, T. Ishii, I. Kudo, Various secretory phospholipase $A_{2}$ enzymes are expressed in rheumatoid arthritis and augment prostaglandin production in cultured synovial cells, FEBS J., 272 (2005) 655-672.

[8] K.F. Scott, M. Sajinovic, J. Hein, S. Nixdorf, P. Galettis, W. Liauw, P. de Souza, Q. Dong, G.G. Graham, P.J. Russell, Emerging roles for phospholipase $A_{2}$ enzymes in cancer, Biochimie, 92 (2010) 601-610.

[9] S. Ayilavarapu, A. Kantarci, G. Fredman, O. Turkoglu, K. Omori, H. Liu, T. Iwata, M. Yagi, H. Hasturk, T.E. Van Dyke, Diabetes-induced oxidative stress is mediated by $\mathrm{Ca}^{2+}{ }^{2}$-independent phospholipase $A_{2}$ in neutrophils, J. Immunol., 184 (2010) 1507-1515.

[10] A. Malhotra, I. Edelman-Novemsky, Y. Xu, H. Plesken, J. Ma, M. Schlame, M.C. Ren, Role of calcium-independent phospholipase $A_{2}$ in the pathogenesis of Barth syndrome, PNAS, 106 (2009) 2337-2341.

[11] L.A. Engel, Z. Jing, D.E. O'Brien, M. Sun, P.T.C. Kotzbauer, Catalytic function of PLA ${ }_{2} G 6$ is impaired by mutations associated with infantile neuroaxonal dystrophy but not dystoniaparkinsonism, PloS One, 5 (2009) e12897.

[12] V.D. Mouchlis, D. Bucher, J.A. McCammon, E.A. Dennis, Membranes serve as allosteric activators of phospholipase $A_{2}$, enabling it to extract, bind, and hydrolyze phospholipid substrates, PNAS, 112 (2015) E516-E525.

[13] D. Bucher, Y.H. Hsu, V.D. Mouchlis, E.A. Dennis, J.A. McCammon, Insertion of the $\mathrm{Ca}^{2+}$ independent phospholipase $A_{2}$ into a phospholipid bilayer via coarse-grained and atomistic molecular dynamics simulations, PLoS Comput. Biol., 9 (2013) e1003156.

[14] Y.-H. Hsu, D. Bucher, J. Cao, S. Li, S.-W. Yang, G. Kokotos, V. Woods, J. McCammon, E. Dennis, Fluoroketone inhibition of $\mathrm{Ca}^{2+}$-independent phospholipase $\mathrm{A}_{2}$ through pinding pocket association defined by hydrogen/deuterium exchange and molecular dynamics, J. Am. Chem. Soc., 135 (2013) 1330-1337. 
[15] E.J. Ackermann, K. Conde-Frieboes, E.A. Dennis, Inhibition of macrophage Ca ${ }^{2+}$ independent phospholipase $A_{2}$ by bromoenol lactone and trifluoromethyl ketones, J. Biol. Chem., 270 (1995) 445-450.

[16] Y.C. Lio, L.J. Reynolds, J. Balsinde, E.A. Dennis, Irreversible inhibition of $\mathrm{Ca}^{2+}$-independent phospholipase $A_{2}$ by methyl arachidonyl fluorophosphonate, Biochim. Biophys. Acta, 1302 (1996) 55-60.

[17] E. Barbayianni, D. Stephens, A. Grkovich, V. Magrioti, Y.-H.H. Hsu, P. Dolatzas, D. Kalogiannidis, E.A. Dennis, G.C. Kokotos, 2-Oxoamide inhibitors of phospholipase $A_{2}$ activity and cellular arachidonate release based on dipeptides and pseudodipeptides, Bioorg. Med. Chem., 17 (2009) 4833-4843.

[18] V. Magrioti, A. Nikolaou, A. Smyrniotou, I. Shah, V. Constantinou-Kokotou, E. Dennis, G. Kokotos, New potent and selective polyfluoroalkyl ketone inhibitors of GVIA calciumindependent phospholipase $A_{2}$, Bioorg. Med. Chem., 21 (2013) 5823-5829.

[19] G. Kokotos, Y.-H. Hsu, J. Burke, C. Baskakis, C. Kokotos, V. Magrioti, E. Dennis, Potent and selective fluoroketone inhibitors of group VIA calcium-independent phospholipase $A_{2}, J$. Med. Chem., 53 (2010) 3602-3610.

[20] L. Pauling, Molecular architecture and biological reactions, Chem. Eng. News, 24 (1946) 1375-1377.

[21] U. Brodbeck, K. Schweikert, R. Gentinetta, Fluorinated aldehydes and ketones acting as quasi-substrate inhibitors of acetylcholinesterase, Biochim. Biophys. Acta, 567 (1979) 357-369.

[22] B.D. Hammock, K.D. Wing, J. McLaughlin, V.M. Lovell, T.C. Sparks, Trifluoromethylketones as possible transition state analog inhibitors of juvenile hormone esterase, Pestic. Biochem. Physiol., 17 (1982) 76-88.

[23] B.D. Hammock, Y.A.I. Abdel-Aal, C.A. Mullin, T.N. Hanzlik, R.M. Roe, Substituted thiotrifluoropropanones as potent selective inhibitors of juvenile hormone esterase, Pestic. Biochem. Physiol., 22 (1984) 209-223.

[24] Y. Abdel-Aal, B. Hammock, Transition state analogs as ligands for affinity purification of juvenile hormone esterase, Science, 233 (1986) 1073-1076.

[25] M.-B.A. Ashour, B.D. Hammock, Substituted trifluoroketones as potent, selective inhibitors of mammalian carboxylesterases, Biochem. Pharmacol., 36 (1987) 1869-1879.

[26] C.E. Wheelock, M.E. Colvin, I. Uemura, M.M. Olmstead, J.R. Sanborn, Y. Nakagawa, D.A. Jones, B.D. Hammock, Use of ab initio calculations to predict the biological potency of carboxylesterase inhibitors, J. Med. Chem., 45 (2002) 5576-5593.

[27] C.E. Wheelock, T.F. Severson, B.D. Hammock, Synthesis of new carboxylesterase inhibitors and evaluation of potency and water solubility, Chem. Res. Toxicol., 14 (2001) 1563-1572.

[28] T.L. Huang, T. Shiotsuki, T. Uematsu, B. Borhan, Structure-activity relationships for substrates and inhibitors of mammalian liver microsomal carboxylesterases, Pharm. Res., 13 (1996) 1495-1500.

[29] M. Wogulis, C.E. Wheelock, S.G. Kamita, A.C. Hinton, P.A. Whetstone, B.D. Hammock, D.K. Wilson, Structural studies of a potent insect maturation inhibitor bound to the juvenile hormone esterase of Manduca sexta, Biochemistry, 45 (2006) 4045-4057.

[30] R.M. Roe, D.D. Anspaugh, K. Venkatesh, A novel geminal diol as a highly specific and stable in vivo inhibitor of insect juvenile hormone esterase, Arch. Insect. Biochem. Physiol., 36 (1997) 165-179. 
[31] P.J. Guthrie, Carbonyl addition reactions: Factors affecting the hydrate-hemiacetal and hemiacetal-acetal equilibrium constants, Canad. J. Chem., 53 (1975) 898-906.

[32] R.M. Wadkins, J.L. Hyatt, C.C. Edwards, L. Tsurkan, M.R. Redinbo, C.E. Wheelock, P.D. Jones, B.D. Hammock, P.M. Potter, Analysis of mammalian carboxylesterase inhibition by trifluoromethylketone-containing compounds, Mol. Pharmacol., 71 (2007) 713-723.

[33] D.A. Six, E. Barbayianni, V. Loukas, V. Constantinou-Kokotou, D. Hadjipavlou-Litina, D. Stephens, A.C. Wong, V. Magrioti, P. Moutevelis-Minakakis, S.F. Baker, E.A. Dennis, G. Kokotos, Structure-activity relationship of 2-oxoamide inhibition of group IVA cytosolic phospholipase $A_{2}$ and group $V$ secreted phospholipase $A_{2}$, J. Med. Chem., 50 (2007) 4222-4235.

[34] H. Yang, M. Mosior, C. Johnson, Y. Chen, E. Dennis, Group-specific assays that distinguish between the four major types of mammalian phospholipase $A_{2}$, Anal. Biochem., 269 (1999) 278-288.

[35] J. Cao, J. Burke, E. Dennis, Using hydrogen/deuterium exchange mass spectrometry to define the specific interactions of the phospholipase $A_{2}$ superfamily with lipid substrates, inhibitors, and membranes, J. Biol. Chem., 288 (2013) 1806-1813.

[36] S. Mehmood, C. Domene, E. Forest, Dynamics of a bacterial multidrug ABC transporter in the inward-and outward-facing conformations, PNAS, 109 (2012) 10832-10836.

[37] G.M. West, E.Y.T. Chien, V. Katritch, J. Gatchalian, Ligand-dependent perturbation of the conformational ensemble for the GPCR $\beta 2$ adrenergic receptor revealed by HDX, Structure, 19 (2011) 1424-1432.

[38] Y.-H. Hsu, J. Burke, S. Li, V. Woods, E. Dennis, Localizing the membrane binding region of Group VIA $\mathrm{Ca}^{2+}$-independent phospholipase $A_{2}$ using peptide amide hydrogen/deuterium exchange mass spectrometry, J. Biol. Chem., 284 (2009) 23652-23661.

[39] J. Burke, Y.-H. Hsu, R. Deems, S. Li, V. Woods, E. Dennis, A phospholipid substrate molecule residing in the membrane surface mediates opening of the lid region in group IVA cytosolic phospholipase $A_{2}$, J. Biol. Chem., 283 (2008) 31227-31236.

[40] J. Burke, A. Babakhani, A. Gorfe, G. Kokotos, S. Li, V. Woods, J. McCammon, E. Dennis, Location of inhibitors bound to group IVA phospholipase $A_{2}$ determined by molecular dynamics and deuterium exchange mass spectrometry, J. Am. Chem. Soc., 131 (2009) 8083-8091.

[41] Induced fit docking protocol 2014, Glide version 6.5, Prime version 3.8, Schrödinger, LLC, New York, NY, 2014., (2014).

[42] J. Phillips, R. Braun, W. Wang, J. Gumbart, E. Tajkhorshid, E. Villa, C. Chipot, R. Skeel, L. Kalé, K. Schulten, Scalable molecular dynamics with NAMD, J. Comput. Chem., 26 (2005) 17811802.

[43] C. Bissantz, B. Kuhn, M. Stahl, A medicinal chemist?]s guide to molecular interactions, Journal of medicinal chemistry, 53 (2010) 5061-5084.

[44] Protein preparation wizard 2014-4; Epik version 2.4, Schrödinger, LLC, New York, NY, 2014; Impact version 5.9, Schrödinger, LLC, New York, NY, 2014; Prime version 3.2, Schrödinger, LLC, New York, NY, 2014., (2014).

[45] Glide, version 6.5, Schrödinger, LLC, New York, NY, 2014., (2014).

[46] C. Baskakis, V. Magrioti, N. Cotton, D. Stephens, V. Constantinou-Kokotou, E.A. Dennis, G. Kokotos, Synthesis of polyfluoro ketones for selective inhibition of human phospholipase $A_{2}$ enzymes, J. Med. Chem., 51 (2008) 8027-8037. 
[47] Y. Chen, E.A. Dennis, Expression and characterization of human group $V$ phospholipase $A_{2}$, Biochim. Biophys. Acta, 1394 (1998) 57-64.

[48] W. Sherman, T. Day, M.P. Jacobson, R.A. Friesner, R. Farid, Novel procedure for modeling ligand/receptor induced fit effects, J. Med. Chem., 49 (2006) 534-553.

[49] Maestro, version 9.9, Schrödinger, LLC, New York, NY, 2014., (2014).

[50] LigPrep, version 3.2, Schrödinger, LLC, New York, NY, 2014., (2014).

[51] Prime, version 3.8, Schrödinger, LLC, New York, NY, 2014., (2014).

[52] R.A. Friesner, R.B. Murphy, M.P. Repasky, L.L. Frye, J.R. Greenwood, T.A. Halgren, P.C. Sanschagrin, D.T. Mainz, Extra precision glide: docking and scoring incorporating a model of hydrophobic enclosure for protein-ligand complexes, J. Med. Chem., 49 (2006) 6177-6196.

[53] K. Vanommeslaeghe, E. Hatcher, C. Acharya, S. Kundu, S. Zhong, J. Shim, E. Darian, O. Guvench, P. Lopes, I. Vorobyov, A.C. Mackerell, CHARMM general force field: A force field for drug-like molecules compatible with the CHARMM all-atom additive biological force fields, J Comput. Chem., 31 (2009) 671-690.

[54] J.B. Klauda, R.M. Venable, J.A. Freites, J.W. O'Connor, D.J. Tobias, C. Mondragon-Ramirez, I. Vorobyov, A.D. MacKerell, R.W.C. Pastor, Update of the CHARMM all-atom additive force field for lipids: validation on six lipid types, J. Phys. Chem. B, 114 (2010) 7830-7843. 


\section{Graphical Abstract}
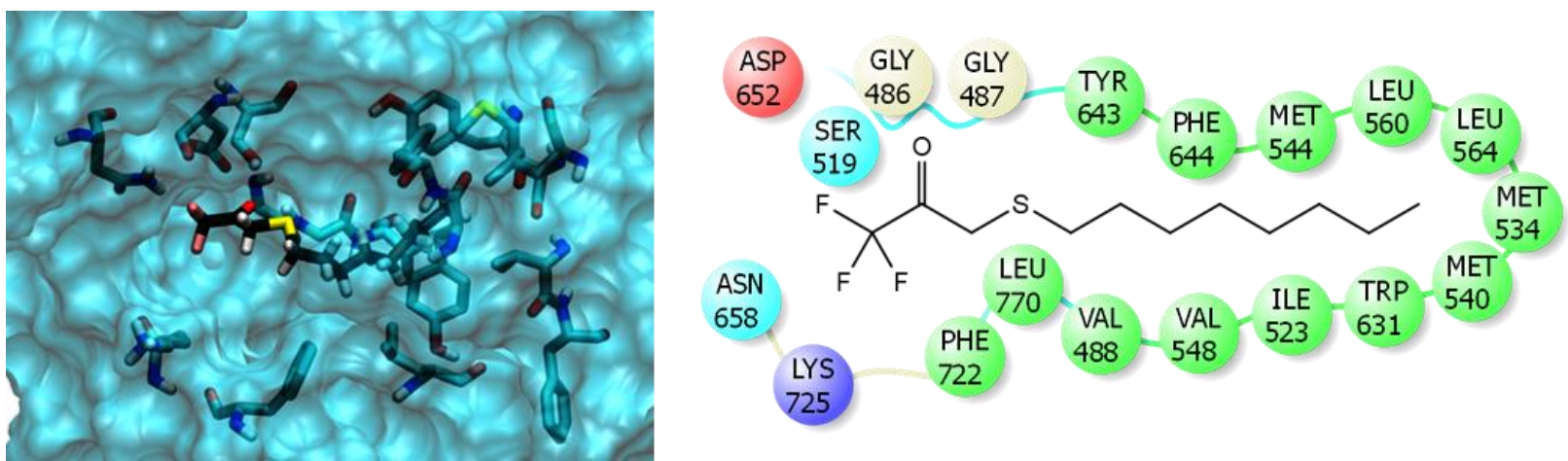